\title{
Association between polymorphisms in folate metabolism genes and maternal risk for Down syndrome: A meta-analysis
}

\author{
YANQING GU \\ Department of Orthopedics, Nanjing First Hospital, Nanjing Medical University, Nanjing, Jiangsu 210006, P.R. China
}

Received July 25, 2016; Accepted April 26, 2017

DOI: $10.3892 /$ mco.2017.1338

\begin{abstract}
Previous studies have focused on the association between polymorphisms of the genes involved in folate metabolism and Down syndrome (DS); however, the results remain inconclusive. The present meta-analysis was conducted to assess the association between RFC-1 A80G/MTR A2756G/CBS 844ins68 polymorphisms and the maternal risk of DS. Published studies were retrieved from PubMed, Embase, China National Knowledge Infrastructure and Chinese Biomedicine databases. Pooled odds ratios (ORs) with 95\% confidence interval (CIs) were calculated using the fixedor random-effects model. Additionally, test of heterogeneity, cumulative meta-analysis, sensitivity analysis and assessment of bias were also performed. Finally, 11, 11 and 6 studies were deemed eligible for meta-analyses of RFC-1 A80G, MTR A2756G and CBS 844ins68, respectively. A significant association between RFC-1 A80G polymorphism and DS risk was observed for $\mathrm{G}$ vs. A (OR=1.19, 95\% CI: 1.004-1.40, $\mathrm{P}=0.04)$ and the recessive model $(\mathrm{OR}=1.28,95 \% \mathrm{CI}: 1.05-1.56, \mathrm{P}=0.01)$. In the stratified analysis by source of control or sample size, a significantly increased risk was observed among hospital-based studies and large-sample groups (>200 subjects), respectively. In addition, the cumulative meta-analysis of the RFC-1 A80G variant revealed a trend toward an association as the amount of data increased. However, for the MTR A2756G and CBS 844 ins68 polymorphisms, no obvious association was found for all genetic models. In summary, the present meta-analysis demonstrated that RFC-1 A80G, but not MTR A2756G or CBS 844ins68, was considered as a maternal risk factor for DS in the offspring.
\end{abstract}

Correspondence to: Dr Yanqing Gu, Department of Orthopedics, Nanjing First Hospital, Nanjing Medical University, 68 Changhong Road, Nanjing, Jiangsu 210006, P.R. China

E-mail: gyqnjmu@163.com; njmugyq@sina.com

Key words: down syndrome, RFC-1 A80G, MTR A2756G, CBS 844ins68, folate metabolism gene polymorphisms

\section{Introduction}

Down syndrome (DS) or trisomy 21 is the most common genetic cause of mental retardation. In $95 \%$ of the cases, DS is caused by an error occurring during meiosis, mainly of maternal origin (1-3). Despite extensive research, the molecular mechanisms underlying the meiotic non-disjunction are poorly understood. However, several studies suggested that genomic DNA hypomethylation may be associated with chromosomal instability and abnormal segregation $(4,5)$. Moreover, impairment of folate metabolism has been causally associated with DNA hypomethylation (6-8). Thus, polymorphisms involved in folate metabolism have been investigated as maternal risk factors for DS. Several key enzymes, including methylenetetrahydrofolate reductase (MTHFR), methionine synthase (MTR) and cystathionine b-synthase (CBS) are involved in the folate metabolic pathway. MTHFR converts tetrahydrofolate to 5,10-methylenetetrahydrofolate, which is vital for nucleic acid metabolism $(9,10)$. C677T and A1298C, two common variants in the MTHFR gene, have been investigated in two previous meta-analyses for their role as maternal risk factors for DS $(11,12)$.

The MTR enzyme, encoded by the MTR gene, catalyzes the remethylation of homocysteine to methionine, which is required for the production of the universal methyl donor adenosylmethionine $(13,14)$. CBS acts in the transsulfuration of homocysteine to cystathionine, playing a critical role in linking the folate and methionine cycles in regulating homocysteine levels $(15,16)$. In addition to the metabolism, folate-transporting proteins, including reduced folate carrier-1 (RFC-1), are also crucial for the maintenance of DNA methylation. RFC-1 is responsible for folate uptake from the jejunum and its subsequent translocation across biological membranes in a variety of cells (17). The A80G polymorphism of the RFC-1 gene has been recently demonstrated to affect plasma folate and homocysteine levels $(18,19)$.

To date, a number of studies have investigated the association between RFC-1 A80G/MTR A2756G/CBS 844ins68 polymorphisms and the risk of DS in the offspring (20-34); however, it remains inconclusive whether these polymorphisms in the mother are causal in determining DS susceptibility in the offspring. Therefore, a meta-analysis of all relevant studies was conducted to quantitatively assess the effect of these three polymorphisms on the risk of DS. 


\section{Data collection methods}

Search strategy. Literature databases, including PubMed, Embase, China National Knowledge Infrastructure (CNKI) and Chinese Biomedicine, were searched for relevant studies (the last search was updated in April 2013). The following search terms were used: ('cystathionine-beta-synthase', 'CBS', 'methionine synthase', 'MTR', 'rs1805087', 'reduced folate carrier 1', or 'RFC 1', 'rs1051266'), ('Down syndrome' or 'trisomy 21'), and (polymorphism OR variant). The search was limited to studies published in English or Chinese language. In addition, a snowball search was conducted to identify additional potentially relevant studies in the references of reviews and retrieved articles.

Inclusion and exclusion criteria. The studies included in the present meta-analysis were required to meet the following criteria: i) RFC-1 A80G or MTR A2756G or CBS 844ins68 polymorphisms and maternal risk for DS; ii) case-control design iii) sufficient maternal genotype data for calculation of odds ratio (OR) with $95 \%$ confidence interval (CI); iv) published in English or Chinese. Studies were excluded for the following reasons: i) Data duplication; ii) no usable maternal genotype data provided; and iii) abstracts, comments and reviews.

Data extraction. The following information was extracted from each study by two investigators independently: Name of the first author, year of publication, source of control subjects, country of origin, ethnicities of the individuals involved, number of cases and controls, and number of genotypes for the three polymorphisms in cases and controls. Disagreements were resolved by discussion between the two investigators.

Statistical analysis. STATA software, version 12 (StataCorp LP, College Station, TX, USA) was used to perform all the statistical analyses. P-values $<0.05$ were considered to indicate statistically significant differences. The distribution of genotypes in the control group of each study was assessed for Hardy-Weinberg equilibrium (HWE) and $\mathrm{P}<0.05$ was considered as significant disequilibrium.

The association between the RFC-1 A80G polymorphism and maternal risk for DS was evaluated using OR and $95 \%$ CI (35) under the codominant, dominant and recessive genetic models, as well as the allele model. For the MTR A2756G and CBS 844ins68 polymorphisms, the four genetic models were also used. In addition, subgroup analysis for the RFC-1 A80G and MTR A2756G polymorphisms was performed based on ethnicity, sample size and source of controls.

All the meta-analyses were evaluated for heterogeneity using the Chi-squared-based $\mathrm{Q}$ test and the $\mathrm{I}^{2}$ test (36). A random-effects model was used when the heterogeneity test result was $\mathrm{P}<0.10$; otherwise, the fixed-effects model was used (37). Moreover, sensitivity analysis was performed to evaluate the stability of the results following sequential removal of each study. Cumulative meta-analyses of associations for each polymorphism were also performed through assortment of studies with publication time. Finally, publication bias was assessed via Egger's test and funnel plots: $\mathrm{P}<0.05$ was considered to indicate statistical significance and the

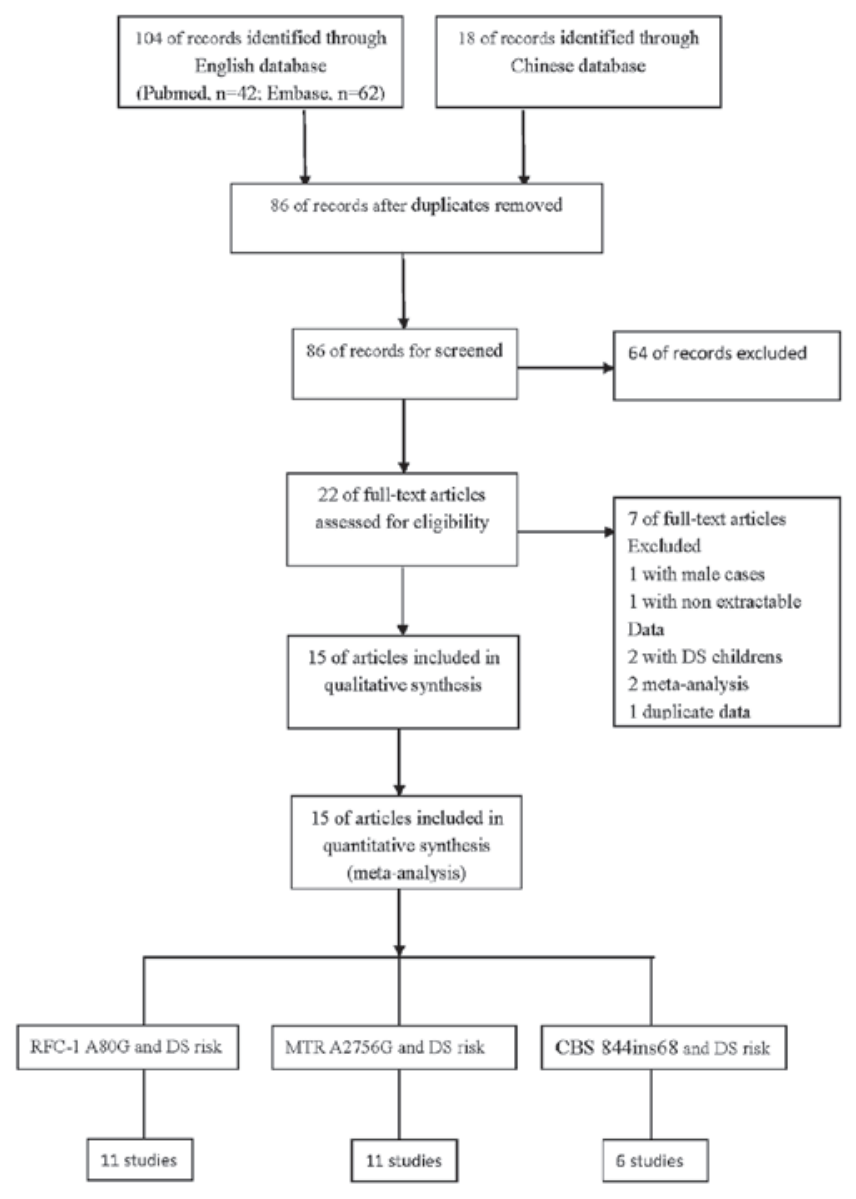

Figure 1. Flow chart of retrieved studies and studies excluded based on specified criteria. DS, Down syndrome.

funnel plot should be asymmetric when there was publication bias (38-40).

\section{Results}

Characteristics of the included studies. A total of 122 titles in the PubMed, Embase, CNKI and Chinese Biomedicine databases were found to be relevant to the search terms. Subsequently, the abstracts and full articles of the retrieved studies were read to assess their eligibility for inclusion in the meta-analysis. Finally, 15 studies investigating the association between any of the RFC-1 A80G, MTR A2756G and CBS 844ins68 polymorphisms and DS were included in the meta-analysis. Among these studies, 2 and 4 studies investigated only MTR A2756G and RFC-1 A80G polymorphisms, respectively; 3 studies included both RFC-1 A80G and MTR A2756G polymorphisms; 2 studies included both MTR A2756G and CBS 844ins68 polymorphisms; and 4 studies investigated RFC-1 A80G, MTR A2756G and CBS 844ins68 polymorphisms. The flow chart of the study selection process is shown in Fig. 1.

There were 11 studies with 2,389 mothers (997 cases and 1,392 controls) for the RFC-1 A80G polymorphism, 11 studies with 2,717 mothers (1,162 cases and 1,555 controls) for the MTR A2756G polymorphism and 6 studies with 1,859 mothers (825 cases and 1,034 controls) for the CBS 


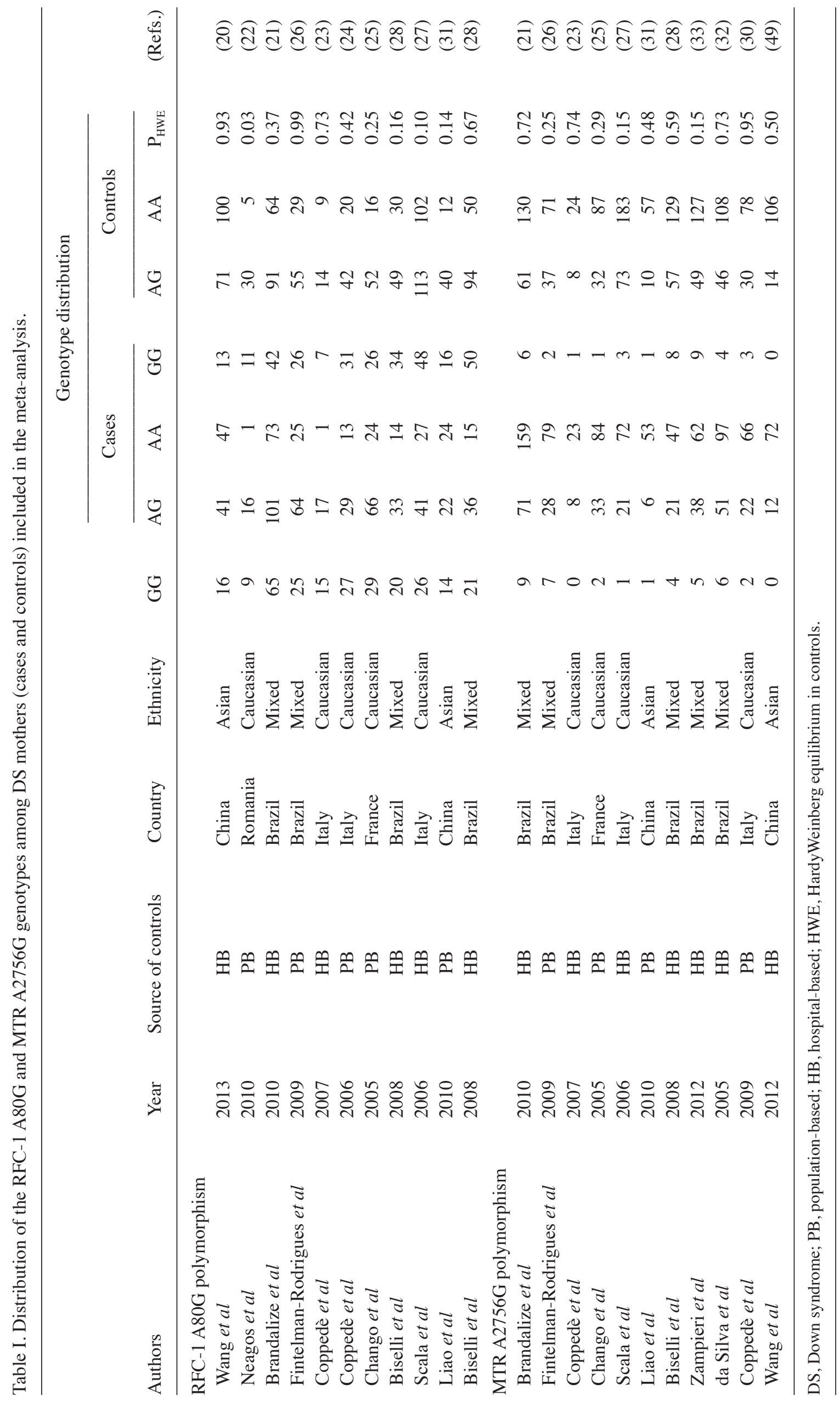


844 ins68 polymorphism. The studies included in the present meta-analysis were conducted on different ethnic populations: 6 studies included a Caucasian population, 3 studies included an Asian population, and 6 studies included a mixed population. The studies were published between 2005 and 2013. Additionally, the distribution of genotypes in the control groups deviated from HWE in the study of Neagos et al (22). The characteristics of all the included studies are summarized in Tables I and II.

RFC-1 A80G polymorphism. A total of 11 case-control studies on the association between RFC-1 A80G polymorphism and maternal risk for DS were included (Table III). The results of the combined analyses revealed a significantly increased maternal DS risk for the RFC-1 A80G polymorphism: G vs. A: $\mathrm{OR}=1.19, \mathrm{P}=0.04$; and recessive model: $\mathrm{OR}=1.28, \mathrm{P}=0.01$ (Fig. 2A). When stratified by ethnicity, a significantly elevated maternal risk for DS was observed among Caucasians in the recessive model $(\mathrm{OR}=1.38, \mathrm{P}=0.046)$, but not among Asians or in the mixed population (Table III).

In the stratified analyses by sample size, a significantly increased risk was observed among large-sample studies ( $>200$ subjects) (G vs. A: OR=1.20, $\mathrm{P}=0.02 ; \mathrm{GG}$ vs. AA: $\mathrm{OR}=1.45, \mathrm{P}=0.02$; dominant model: $\mathrm{OR}=1.25, \mathrm{P}=0.04$; and recessive model: $\mathrm{OR}=1.29, \mathrm{P}=0.03$ ); however, an increased risk was not observed in small-sample studies ( $\leq 200$ subjects) (Table III). Moreover, when subgroup analysis was performed by source of controls, a significantly increased DS risk was found among hospital-based (HB) controls (G vs. A: $\mathrm{OR}=1.34$, $\mathrm{P}=0.001$; GG vs. AA: $\mathrm{OR}=1.76, \mathrm{P}<0.001$; dominant model: $\mathrm{OR}=1.37, \mathrm{P}=0.02$; recessive model: $\mathrm{OR}=1.47, \mathrm{P}<0.001$ ), but not among population-based (PB) controls. Interestingly, a statistical correlation between homozygotes and the dominant model, observed in large-sample and HB control groups, was not found in the overall comparison.

MTR A2756G and CBS 844ins68 polymorphisms. The analysis of the MTR A2756G polymorphism and its association with maternal DS risk revealed that the fixed-effects pooled OR for the recessive model: GG vs. GA+AA was non-significant: $\mathrm{OR}=1.24, \mathrm{P}=0.37$. (Fig. 2B) Additionally, the allele, dominant and codominant models revealed no significant association. Finally, for the CBS 844ins68 polymorphism, no statistically significant association with maternal DS risk was observed in any of the comparisons (Table IV). Fig. 2C shows the recessive model comparison for the CBS 844ins68 polymorphism. Additionally, a subgroup analysis by ethnicity, source of controls and sample size was performed for the two polymorphisms; however, no significant associations were observed.

Heterogeneity analysis. There was significant heterogeneity in the three genetic models for the RFC-1 A80G polymorphism: $\mathrm{G}$ vs. $\mathrm{A}: \mathrm{P}=0.04$, $\mathrm{GG}$ vs. $\mathrm{AA}: \mathrm{P}=0.03$ and $\mathrm{GG}+\mathrm{GA}$ vs. $\mathrm{AA}$ : $\mathrm{P}=0.01$ (Table III). The source of heterogeneity was assessed by ethnicity (Caucasian/Asian/mixed), publication year (prior to or during 2009/after 2009), source of controls (HB/PB) and sample size ( $\leq 200 />200$ subjects). The subgroup analyses revealed removed heterogeneities in several subgroups, including the Caucasian and mixed population, and the 


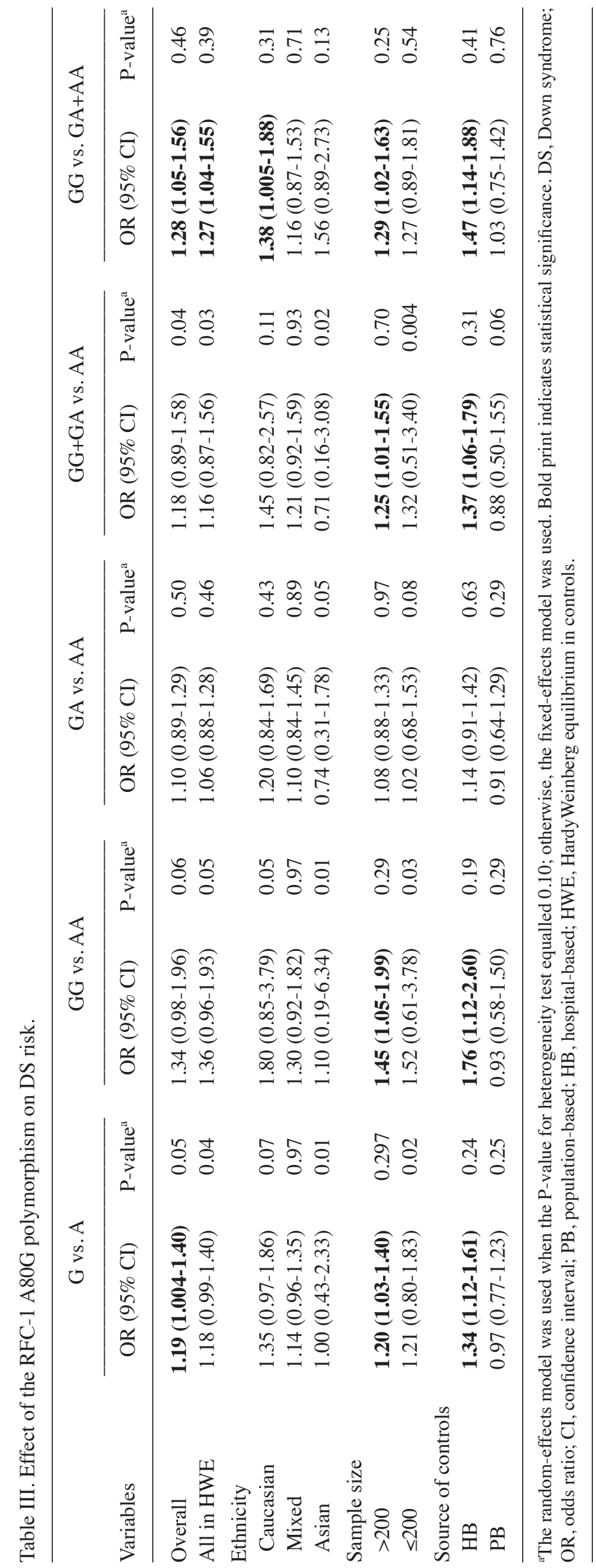


A study

ID

OR $(95 \% \mathrm{Cl}) \quad$ Weight

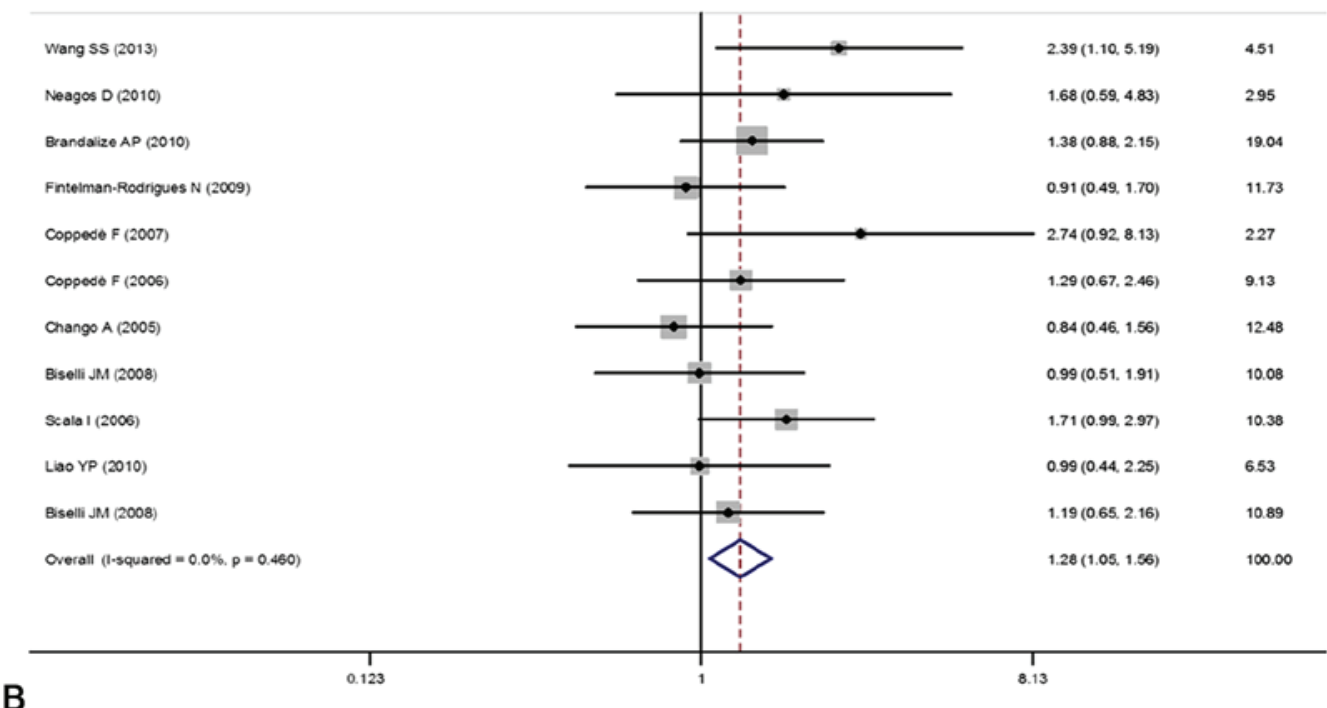

Study

ID

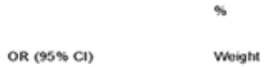

Brandalize AP (2010)

Fntelman-Rodrigues $N$ (2009)

Coppede $F(2007)$

Chango A (2005)

Scals 1 (2006)

Liao YP (2010)

Bselli JM 2008)

Zampieri BL (2012)

da Siva LR (2005)

coppeso F (2009)

Wang XD (2012)

Overall (1-squared $=0.0 \%, p=0.965)$

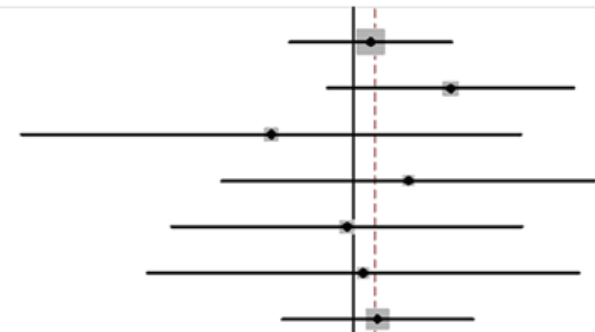

$1.25(0.44,3.56)$

$3.53(0.72,17.39) \quad 6.40$

$0.34(0.01,8.76) \quad 4.79$

$203(0.18,2274) \quad 328$

$0.92(0.09,8.93) \quad 5.29$

$1.14(0.07 .18 .56) \quad 3.09$

$1.37(0.40 .4 .69) \quad 13.69$

$0.98(0.32,3.00) \quad 20.78$

$1.56(0.43,5.64) \quad 12.70$

$0.82(0.13,5.01) \quad 8.79$

(Excluded) $\quad 0.00$

C

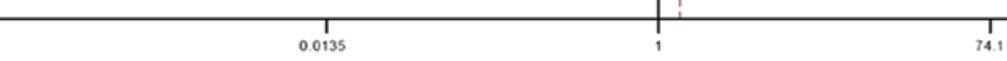

$132(0.82,2.11) \quad 10000$

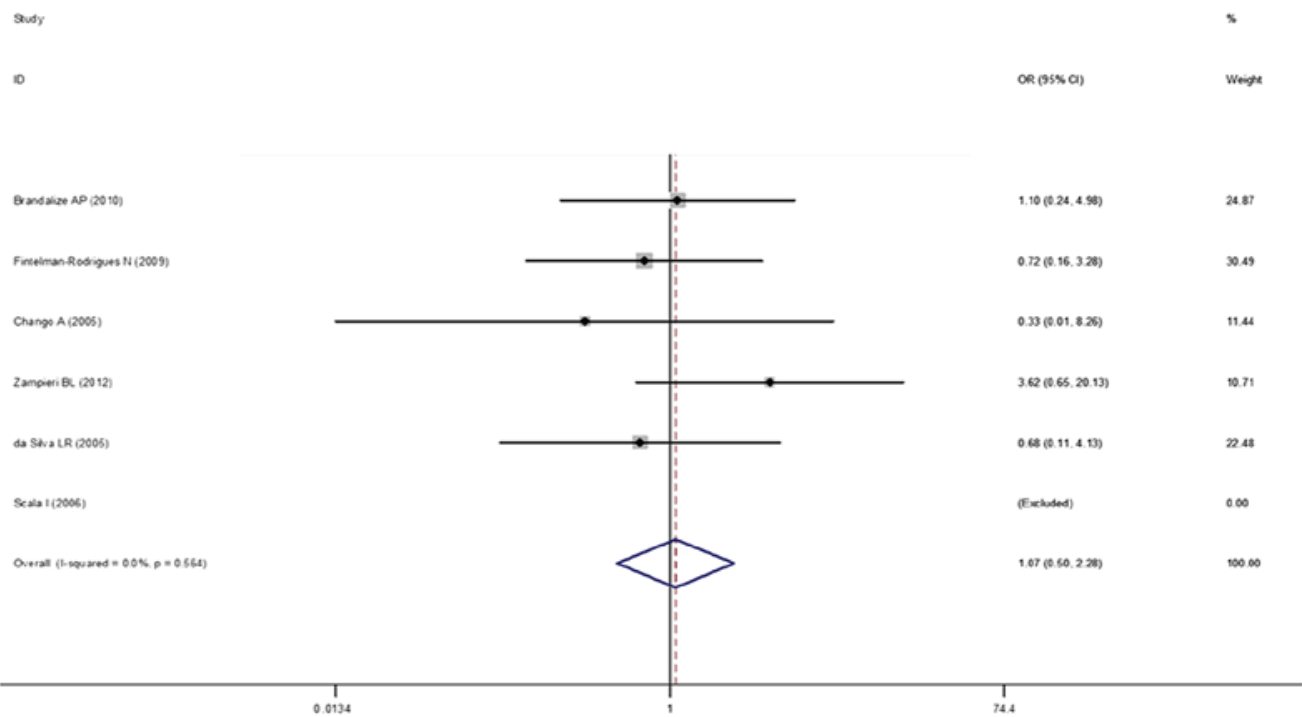

Figure 2. Forest plot of the association between the risk of Down syndrome and the three maternal polymorphisms. (A) RFC-1 A80G polymorphism (GG vs. GA+AA), (B) MTR A2756G polymorphism (GG vs. GA+AA) and (C) CBS 844ins68 polymorphism (ii vs. iw+ww). OR, odds ratio; CI, confidence interval; w, wide-type allele; i, allele with the 68 bp insertion. 


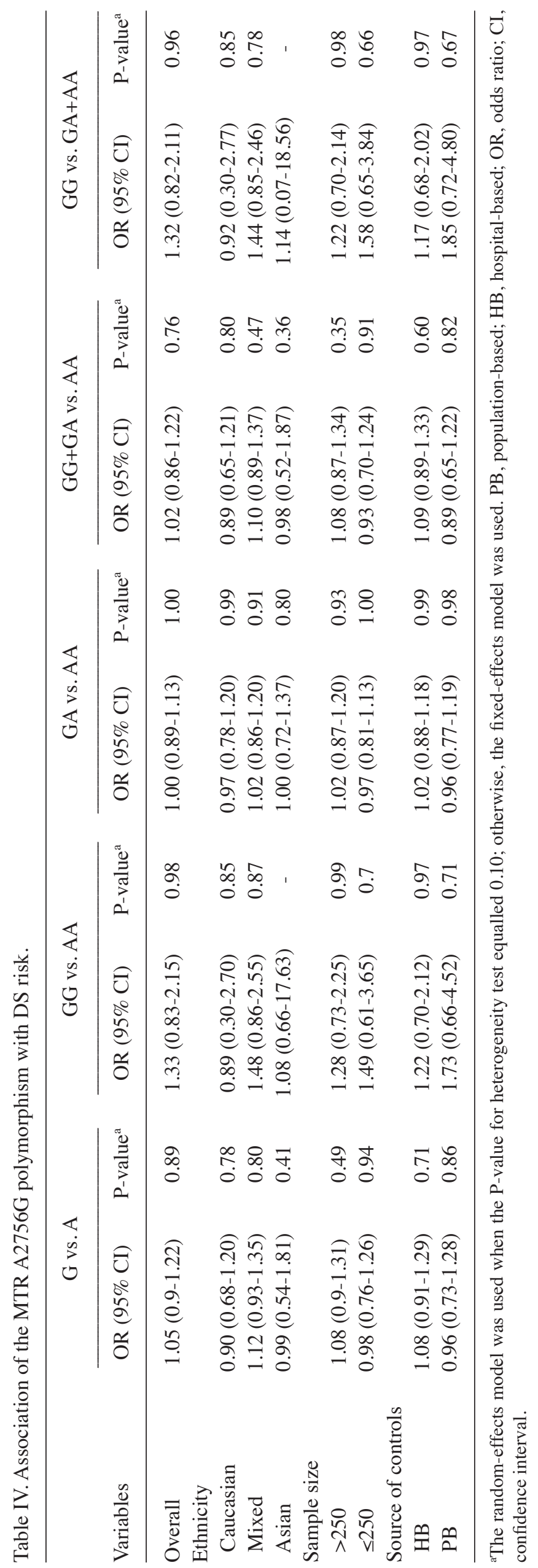

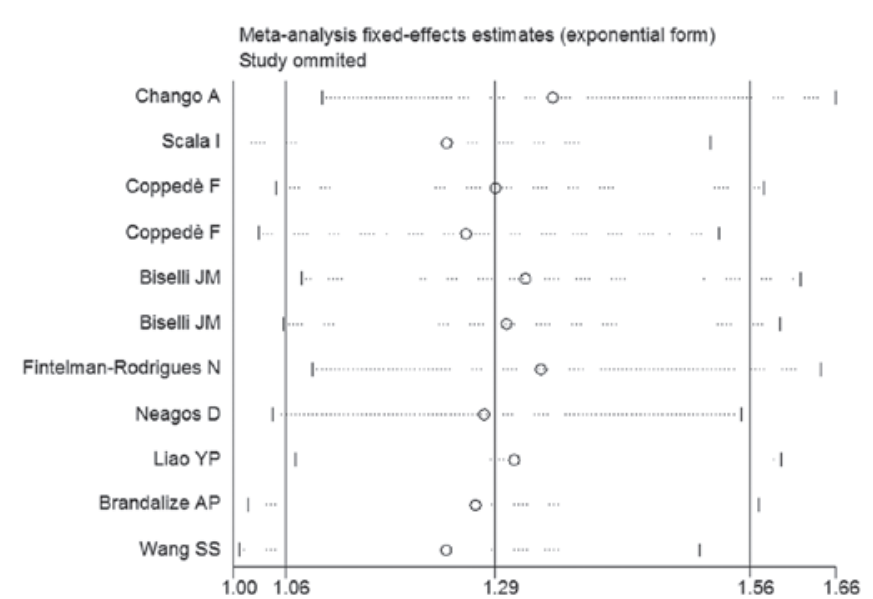

Figure 3. Sensitivity analysis on the association between RFC-1 A80G and the risk of Down syndrome in the recessive model. The results were computed by omitting each study.

large-sample group. However, meta-regression analyses did not reveal any sources contributing to the substantial heterogeneity. For the MTRR A2756G and CBS 844ins68 polymorphisms and their association with DS risk, no statistically significant heterogeneity was observed in any of the genetic models.

Sensitivity analysis. Sensitivity analysis was performed following sequential removal of each eligible study. As regards the association of the RFC-1 A80G polymorphism with maternal DS risk, no single study qualitatively affected the pooled ORs, indicating that the results of the meta-analysis were highly stable (Fig. 3). There were 2 studies that departed from HWE for the CBS 844ins68 polymorphism but, when they were excluded, the pooled OR remained unaffected (Table V).

Cumulative meta-analysis. Cumulative meta-analyses of the 3 associations were also conducted via the assortment of studies by publication time. The results of the association of the RFC-1A80G polymorphism with DS risk for cumulative meta-analysis in chronological order are shown in Fig. 4. The pooled ORs tended to be stable, and the associations exhibited a trend towards significance with the accumulation of more data over time.

Publication bias. Egger's test and funnel plots were applied to evaluate potential publication bias for the RFC-1 A80G, MTR A2756G and CBS 844ins68 polymorphisms (Table VI). The results revealed no evidence of publication bias. The funnel plot investigating the maternal RFC-1 A80G/MTR A2756G/CBS 844 ins68 polymorphisms and DS risk is shown in Fig. 5.

\section{Discussion}

The folate metabolism pathway plays an important role in DNA methylation, DNA synthesis and cell division (41). Recent meta-analyses suggested that the MTHFR C667T polymorphism of the folate metabolism gene may be associated with an increased occurrence of congenital heart defects or neural tube defects $(42,43)$. Abnormal folate metabolism 
and variants of key enzymes in the folate cycle have been described as possible risk factors for DS through impairing DNA methylation (44-46). A80G polymorphism in the RFC-1 gene may impair folate membrane transport. Recently, several studies were performed to evaluate the effects of the RFC-1 A80G/MTR A2756G/CBS 844ins68 variant on the risk of DS, but the results were inconclusive. For example, Brandalize et al (21) and Wang et al (20) reported opposite results on the correlation between the RFC-1 A80G polymorphism and DS risk.

In the present meta-analysis, a significant correlation was observed between the RFC-1 A80G polymorphism and maternal DS risk. A significantly increased DS risk was observed in $\mathrm{G}$ vs. A and in the recessive model $(\mathrm{OR}=1.19$, $\mathrm{P}=0.04$; and $\mathrm{OR}=1.28, \mathrm{P}=0.01$, respectively). However, for MTR A2756G/CBS 844ins68 variants, no significant association between the two polymorphisms and maternal DS risk was observed; the available evidence did not support these two polymorphisms as independent risk factors for DS, which may be attributed to the fact that the interaction of gene polymorphisms rather than any individual polymorphism may be a major determinant of disease risk (47).

In the subgroup analysis stratified by ethnicity, a significant increased DS risk was not observed among Caucasians, Asians and the mixed population for the MTR A2756G and CBS 844ins68 polymorphisms. Thus, the data of the present study suggest that the interactions of these polymorphisms with ethnic variations may not significantly affect DS risk. However, in the recessive model for the RFC-1 A80G variant, a statistically significant increased risk was observed (OR $=1.38$, $\mathrm{P}=0.046$ ). Further large-scale studies are required to investigate the possible ethnic differences in the association of the RFC-1 A80G polymorphism with DS risk.

In the stratification analysis by study sample size for the RFC-1 A80G variant, a significantly increased DS risk was only observed in large-sample studies ( $>200$ subjects). In addition, a statistically significant correlation was found between the RFC-1 A80G polymorphism and DS risk for the dominant model and homozygotes, which was not observed in the overall study sample. These results indicate that large-sample studies may offer quite different outcomes compared with small-sample studies, which is possibly due to the fact that small and underpowered studies may be unable to identify true genetic associations (48). Therefore, the use of a proper, large-sample study is crucial for reducing biases in such genotype association studies. In the subgroup analysis stratified by source of controls, a significantly increased DS risk was observed in the HB groups, but not among PB groups. However, HB controls may not always be truly representative of the general population, particularly when the polymorphisms under investigation are expected to affect disease conditions, which may be observed in the HB controls, indicating the presence of possible selection bias. Thus, further studies using proper controls with strict matching criteria are crucial for reducing such selection biases.

In the present meta-analysis, all the available relevant publications in English and Chinese were searched. Furthermore, the Q-test and $\mathrm{I}^{2}$ statistics were applied to test the significance of heterogeneity. Heterogeneity was observed in three genetic models; subsequently, meta-regression analysis and subgroup 
Table VI. Egger's publication bias test for RFC-1 A80G, MTR A2756G and CBS 844ins68 polymorphisms.

\begin{tabular}{|c|c|c|c|c|c|}
\hline Comparisons & Coefficient & Standard error & $\mathrm{t}$ & $\mathrm{P}>|\mathrm{t}|$ & $95 \% \mathrm{CI}$ \\
\hline \multicolumn{6}{|c|}{ RFC-1 A80G polymorphism } \\
\hline G vs. A & 0.81 & 1.63 & 0.50 & 0.63 & $-2.88,4.51$ \\
\hline GG vs. AA & 1.08 & 1.20 & 0.90 & 0.39 & $-1.63,3.78$ \\
\hline GA vs. AA & 0.79 & .74 & 1.07 & 0.314 & $-0.89,2.47$ \\
\hline GG+GA vs. AA & 0.75 & 1.15 & 0.65 & 0.53 & $-1.84,3.34$ \\
\hline GG vs. GA+AA & 1.05 & 1.26 & 0.83 & 0.43 & $-1.81,3.92$ \\
\hline \multicolumn{6}{|c|}{ MTR A2756G polymorphism } \\
\hline G vs. A & -0.87 & 0.73 & -1.19 & 0.27 & $-2.52,0.79$ \\
\hline GG vs. AA & -0.39 & 0.51 & -0.77 & 0.46 & $-1.58,0.79$ \\
\hline GA vs. AA & -.122 & 0.59 & -0.21 & 0.84 & $-1.45,1.21$ \\
\hline GG+GA vs. AA & -0.73 & 0.94 & -0.78 & 0.46 & $-2.86,1.40$ \\
\hline GG vs. GA+AA & -0.27 & 0.56 & -0.49 & 0.64 & $-1.56,1.02$ \\
\hline \multicolumn{6}{|c|}{ CBS 844ins68 polymorphism } \\
\hline i vs. w & -2.37 & 1.04 & -2.28 & 0.08 & $-5.26,0.51$ \\
\hline ii vs. ww & -1.25 & 1.74 & -0.72 & 0.52 & $-6.78,4.28$ \\
\hline iw vs. ww & -2.13 & 2.07 & -1.03 & 0.36 & $-7.88,3.62$ \\
\hline iitiw vs. ww & -2.31 & 1.58 & -1.46 & 0.22 & $-6.72,2.09$ \\
\hline ii vs. iw+ww & -1.16 & 1.85 & -0.63 & 0.58 & $-7.06,4.74$ \\
\hline
\end{tabular}

CI, confidence interval; w, wide-type allele; i, allele with the 68bp insertion.

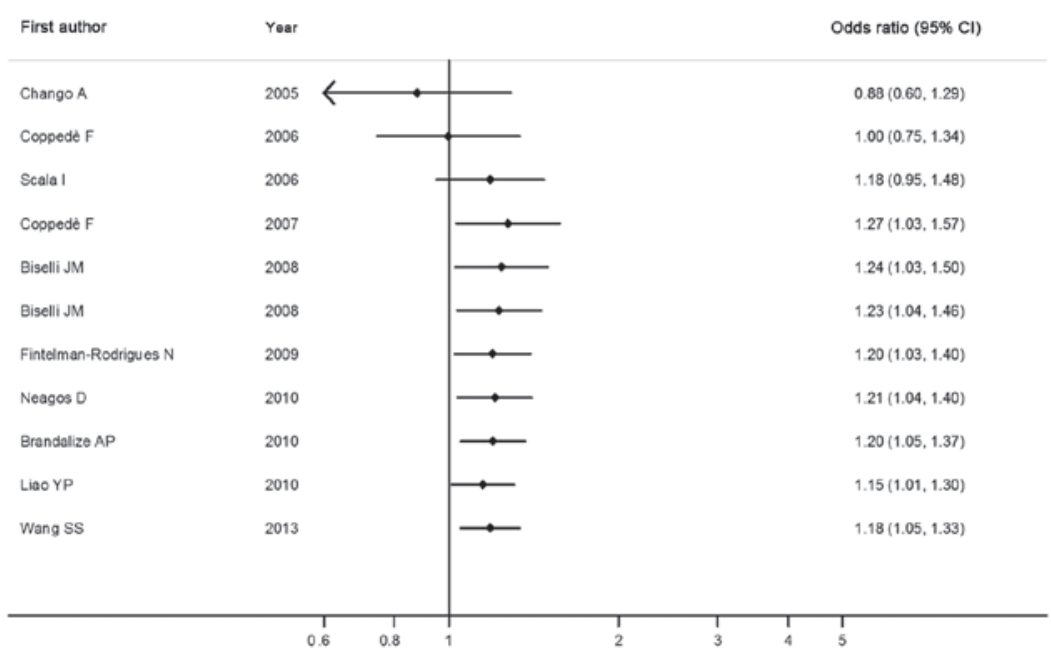

Figure 4. Cumulative meta-analysis: Pooled OR with the corresponding 95\% CI at the end of each year; information is shown for RFC-1 A80G (G vs. A). OR, odds ratio; CI, confidence interval.
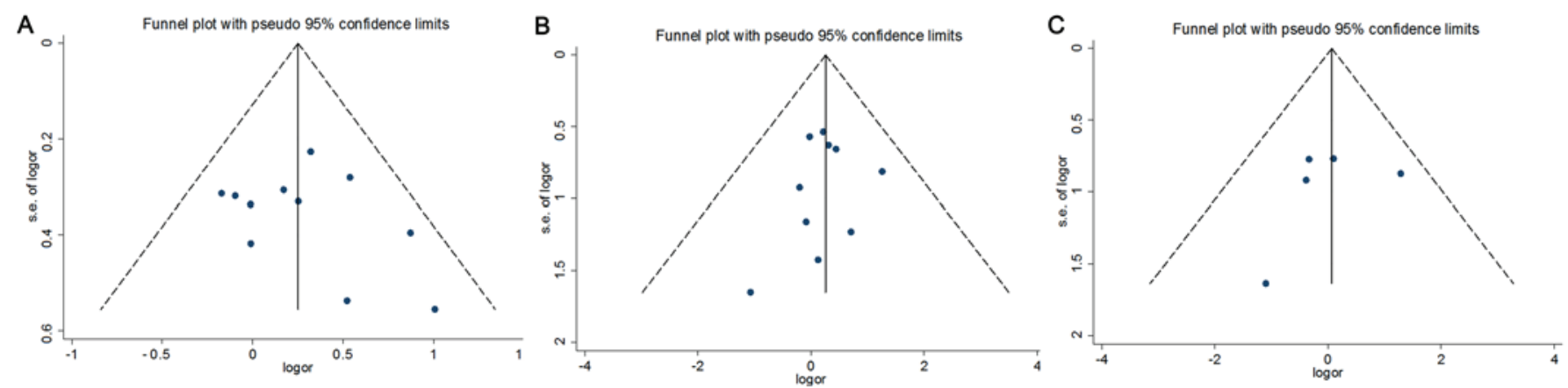

Figure 5. Funnel plot analysis for odds ratios of the dominant model for the three polymorphisms. (A) RFC-1 A80G, (B) MTR A2756G and (C) CBS 844ins68. 
analyses were conducted. As a result, heterogeneities were removed in the subgroup analysis on the Caucasian and mixed populations, as well as on the large-sample group. Moreover, publication bias, an important factor considered in meta-analyses, was evaluated by funnel plots and Egger's tests. Neither the shape of the funnel plots nor the statistical results demonstrated publication bias, indicating the robustness and reliability of the results.

There were certain limitations to this meta-analysis: First, folate metabolism is complex and involves several regulatory mechanisms. Coppedè et al (24) indicated that the presence of both RFC-1 80GG and MTHFR 677GG was associated with a significantly increased risk of a DS offspring $(\mathrm{OR}=6,95 \% \mathrm{CI}$ : 1.00-35.9), which was also reported by other studies $(26,27)$. However, such gene-gene interactions were not addressed in the present meta-analysis due to the lack of sufficient data. Second, a subgroup analysis by maternal age was not performed in the present meta-analysis. Maternal age is the major risk factor for DS, although several children with DS are born to mothers aged $<35$ years. Recently, Scala et al (27) demonstrated that the $80 \mathrm{G}$ allele may increase DS risk in mothers aged $>34$ years at conception. However, only two studies in the present meta-analysis reported the association between genetic polymorphisms and maternal age. Third, the primary articles included in the present meta-analysis only provided data on Caucasian, Asian and mixed ethnicities. The majority of the studies included Caucasians and mixed populations, whereas data on other ethnicities were not available. Finally, although there was no publication bias observed in the funnel plot and Egger's test, selection bias may have occurred, as only studies in English or Chinese were selected.

In summary, the present meta-analysis suggests that the RFC-1 A80G polymorphism may be a maternal genetic risk for DS, particularly in the large-sample and HB control groups. However, there was no evidence of an association between the MTR A2756G/CBS 844ins68 polymorphism and maternal DS risk. Further well-designed large studies are required to investigate gene-environment interactions, gene-maternal age interactions and combinations of gene polymorphisms.

\section{References}

1. Antonarakis SE, Petersen MB, McInnis MG, Adelsberger PA, Schinzel AA, Binkert F, Pangalos C, Raoul O, Slaugenhaupt SA, Hafez M, et al: The meiotic stage of nondisjunction in trisomy 21: Determination by using DNA polymorphisms. Am J Hum Genet 50: 544-550, 1992.

2. Jyothy A, Kumar KS, Mallikarjuna GN, Babu Rao V, Uma Devi B, Sujatha M and Reddy PP: Parental age and the origin of extra chromosome 21 in Down syndrome. J Hum Genet 46: 347-350, 2001.

3. Oliver TR, Feingold E, Yu K, Cheung V, Tinker S, Yadav-Shah M, Masse N and Sherman SL: New insights into human nondisjunction of chromosome 21 in oocytes. PLoS Genet 4: e1000033, 2008.

4. Chen RZ, Pettersson U, Beard C, Jackson-Grusby L and Jaenisch R: DNA hypomethylation leads to elevated mutation rates. Nature 395: 89-93, 1998.

5. Fenech M: Micronutrients and genomic stability: A new paradigm for recommended dietary allowances (RDAs). Food Chem Toxicol 40: 1113-1117, 2002.

6. Liu JJ and Ward RL: Folate and one-carbon metabolism and its impact on aberrant DNA methylation in cancer. Adv Genet 71: 79-121, 2010.

7. McKay JA, Groom A, Potter C, Coneyworth LJ, Ford D, Mathers JC and Relton CL: Genetic and non-genetic influences during pregnancy on infant global and site specific DNA methylation: Role for folate gene variants and vitamin B12. PLoS One 7: e33290, 2012.
8. James SJ, Melnyk S, Jernigan S, Pavliv O, Trusty T, Lehman S, Seidel L, Gaylor DW and Cleves MA: A functional polymorphism in the reduced folate carrier gene and DNA hypomethylation in mothers of children with autism. Am J Med Genet B Neuropsychiatr Genet 153B: 1209-1220, 2010.

9. Bailey LB: New standard for dietary folate intake in pregnant women. Am J Clin Nutr 71 (5 Suppl): 1304S-1307S, 2000.

10. Goyette P, Sumner JS, Milos R, Duncan AM, Rosenblatt DS, Matthews RG and Rozen R: Human methylenetetrahydrofolate reductase: Isolation of cDNA mapping and mutation identification. Nat Genet 7: 551, 1994.

11. Wu X, Wang X, Chan Y, Jia S, Luo Y and Tang W: Folate metabolism gene polymorphisms MTHFR C677T and A1298C and risk for Down syndrome offspring: A meta-analysis. Eur J Obstet Gynecol Reprod Biol 167: 154-159, 2013.

12. Zintzaras E: Maternal gene polymorphisms involved in folate metabolism and risk of Down syndrome offspring: A meta-analysis. J Hum Genet 52: 943-953, 2007.

13. van der Put NM, van der Molen EF, Kluijtmans LA, Heil SG, Trijbels JM, Eskes TK, Van Oppenraaij-Emmerzaal D, Banerjee R and Blom HJ: Sequence analysis of the coding region of human methionine synthase: Relevance to hyperhomocysteinaemia in neural-tube defects and vascular disease. QJM 90: 511-517, 1997.

14. Leclerc D, Campeau E, Goyette P, Adjalla CE, Christensen B, Ross M, Eydoux P, Rosenblatt DS, Rozen R and Gravel RA: Human methionine synthase: cDNA cloning and identification of mutations in patients of the cblG complementation group of folate/cobalamin disorders. Hum Mol Genet 5: 1867-1874, 1996.

15. Tsai MY, Bignell M, Schwichtenberg K and Hanson NQ: High prevalence of a mutation in the cystathionine beta-synthase gene. Am J Hum Genet 59: 1262-1267, 1996.

16. Zhang J, Handy DE, Wang Y, Bouchard G, Selhub J, Loscalzo J and Carey MC: Hyperhomocysteinemia from trimethylation of hepatic phosphatidylethanolamine during cholesterol cholelithogenesis in inbred mice. Hepatology 54: 697-706, 2011.

17. Lopreato FR, Stabler SP, Carvalho FR, Hirata RD, Hirata MH, Robi DL, Sampaio-Neto LF, Allen RH and Guerra-Shinohara EM: Relationships between gene polymorphisms of folate-related proteins and vitamins and metabolites in pregnant women and neonates. Clin Chim Acta 398: 134-139, 2008.

18. Sukla KK and Raman R: Association of MTHFR and RFC1 gene polymorphism with hyperhomocysteinemia and its modulation by vitamin B12 and folic acid in an Indian population. Eur J Clin Nutr 66: 111-118, 2012.

19. Chango A, Emery-Fillon N, de Courcy GP, Lambert D, Pfister M, Rosenblatt DS and Nicolas JP: A polymorphism (80G- $>$ A) in the reduced folate carrier gene and its associations with folate status and homocysteinemia. Mol Genet Metab 70: 310-315, 2000.

20. Wang SS, Wang C, Qiao FY, Lv JJ and Feng L: Polymorphisms in genes RFC-1/CBS as maternal risk factors for Down syndrome in China. Arch Gynecol Obstet 288: 273-277, 2013.

21. Brandalize AP, Bandinelli E, Dos Santos PA and Schüler-Faccini L: Maternal gene polymorphisms involved in folate metabolism as risk factors for Down syndrome offspring in Southern Brazil. Dis Markers 29: 95-101, 2010.

22. Neagos D, Cretu R, Tutulan-Cunita A, Stoian V and Bohiltea LC: RFC-1 Gene Polymorphism and the Risk of Down Syndrome in Romanian Population. Maedica (Buchar) 5: 280-285, 2010.

23. Coppedè F, Colognato R, Bonelli A, Astrea G,Bargagna S, Siciliano G and Migliore L: Polymorphisms in folate and homocysteine metabolizing genes and chromosome damage in mothers of Down syndrome children. Am J Med Genet A 143A: 2006-2015, 2007.

24. Coppedè F, Marini G, Bargagna S, Stuppia L, Minichilli F, Fontana I, Colognato R, Astrea G, Palka G and Migliore L: Folate gene polymorphisms and the risk of Down syndrome pregnancies in young Italian women. Am J Med Genet A 140: 1083-1091, 2006.

25. Chango A, Fillon-Emery N, Mircher C, Bléhaut H, Lambert D, Herbeth B, James SJ, Réthoré MO and Nicolas JP: No association between common polymorphisms in genes of folate and homocysteine metabolism and the risk of Down's syndrome among French mothers. Br J Nutr 94: 166-169, 2005.

26. Fintelman-Rodrigues N, Corrêa JC, Santos JM, Pimentel MM and Santos-Rebouças CB: Investigation of CBS, MTR, RFC-1 and TC polymorphisms as maternal risk factors for Down syndrome. Dis Markers 26: 155-161, 2009.

27. Scala I, Granese B, Sellitto M, Salomé S, Sammartino A, Pepe A, Mastroiacovo P, Sebastio G and Andria G: Analysis of seven maternal polymorphisms of genes involved in homocysteine/folate metabolism and risk of Down syndrome offspring. Genet Med 8: 409-416, 2006. 
28. Biselli JM, Brumati D, Frigeri VF, Zampieri BL, Goloni-Bertollo EM and Pavarino-Bertelli EC: A80G polymorphism of reduced folate carrier 1 (RFC1) and C776G polymorphism of transcobalamin 2(TC2) genes in Down's syndrome etiology. Sao Paulo Med J 126: 329-332, 2008.

29. Biselli JM, Goloni-Bertollo EM, Zampieri BL, Haddad R, Eberlin MN and Pavarino-Bertelli EC: Genetic polymorphisms involved in folate metabolism and elevated plasma concentrations of homocysteine: Maternal risk factors for Down syndrome in Brazil. Genet Mol Res 7: 33-42, 2008.

30. Coppedè F, Grossi E, Migheli F and Migliore L: Polymorphisms in folate-metabolizing genes, chromosome damage, and risk of Down syndrome in Italian women: Identification of key factors using artificial neural networks. BMC Med Genomics 3: 42, 2010

31. Liao YP, Bao MS, Liu CQ, Liu H and Zhang D: Folate gene polymorphism and the risk of Down syndrome pregnancies in young Chinese women. Yi Chuan 32: 461-466, 2010 (In Chinese).

32. da Silva LR, Vergani N, Galdieri Lde C, Ribeiro Porto MP, Longhitano SB, Brunoni D, D'Almeida V and Alvarez Perez AB Relationship between polymorphisms in genes involved in homocysteine metabolism and maternal risk for Down syndrome in Brazil. Am J Med Genet A 135: 263-267, 2005.

33. Zampieri BL, Biselli JM, Goloni-Bertollo EM, Vannucchi H, Carvalho VM, Cordeiro JA and Pavarino EC: Maternal risk for Down syndrome is modulated by genes involved in folate metabolism. Dis Markers 32: 73-81, 2012.

34. Coppedè F, Migheli F, Bargagna S, Siciliano G, Antonucci I, Stuppia L, Palka G and Migliore L: Association of maternal polymorphisms in folate metabolizing genes with chromosome damage and risk of Down syndrome offspring. Neurosci Lett 449: 15-19, 2009

35. Zintzaras E, Doxani C, Koufakis T, Kastanis A, Rodopoulou P and Karachalios T: Synopsis and meta-analysis of genetic association studies in osteoporosis for the focal adhesion family genes: The CUMAGAS-OSTEOporosis information system. BMC Med 9: 9,2011.

36. Higgins JP and Thompson SG: Quantifying heterogeneity in a meta-analysis. Stat Med 21: 1539-1558, 2002

37. DerSimonian R and Laird N: Meta-analysis in clinical trials. Control Clin Trials 7: 177-188, 1986.

38. Macaskill P, Walter SD and Irwig L: A comparison of methods to detect publication bias in meta-analysis. Stat Med 20: 641-654, 2001.

39. Begg CB and Mazumdar M: Operating characteristics of a rank correlation test for publication bias. Biometrics 50: 1088-1101, 1994.

40. Egger M, Davey Smith G, Schneider M and Minder C: Bias in meta-analysis detected by a simple, graphical test. BMJ 315: 629-634, 1997.

41. Morrison K, Papapetrou C, Hol FA, Mariman EC, Lynch SA, Burn J and Edwards YH: Susceptibility to spina bifida; an association study of five candidate genes. Ann Hum Genet 62: 379-396, 1998
42. Yan L, Zhao L, Long Y, Zou P, Ji G, Gu A and Zhao P: Association of the maternal MTHFR C677T polymorphism with susceptibility to neural tube defects in offsprings: Evidence from 25 case-control studies. PLoS One 7: e41689, 2012.

43. Yin M, Dong L, Zheng J, Zhang H, Liu J and Xu Z: Meta analysis of the association between MTHFR C677T polymorphism and the risk of congenital heart defects. Ann Hum Genet 76: 9-16, 2012.

44. Marucci GH, Zampieri BL, Biselli JM, Valentin S, Bertollo EM, Eberlin MN, Haddad R, Riccio MF, Vannucchi H, Carvalho VM and Pavarino EC: Polymorphism C1420T of Serine hydroxymethyltransferase gene on maternal risk for Down syndrome. Mol Biol Rep 39: 2561-2566, 2012

45. Costa-Lima MA, Amorim MR and Orioli IM: Association of methylenetetrahydrofolate reductase gene $677 \mathrm{C}>\mathrm{T}$ polymorphism and Down syndrome. Mol Biol Rep 40: 2115-2125, 2013.

46. Biselli JM, Zampieri BL, Goloni-Bertollo EM, Haddad R, Fonseca MF, Eberlin MN, Vannucchi H, Carvalho VM and Pavarino EC: Genetic polymorphisms modulate the folate metabolism of Brazilian individuals with Down syndrome. Mol Biol Rep 39: 9277-9284, 2012.

47. Hobbs CA, Cleves MA, Lauer RM, Burns TL and James SJ: Preferential transmission of the MTHFR $677 \mathrm{~T}$ allele to infants with Down syndrome: Implications for a survival advantage. Am J Med Genet 113: 9-14, 2002.

48. Hindorff LA, Sethupathy P, Junkins HA, Ramos EM, Mehta JP, Collins FS and Manolio TA: Potential etiologic and functional implications of genome-wide association loci for human diseases and traits. Proc Natl Acad Sci USA 106: 9362-9367, 2009.

49. Wang XD, Gong J, Wu Y, Cao SY, Yang SY, Li MR, et al: Genetic polymorphisms involved in folate metabolism as maternal risk factors for Down syndrome. Chin J Birth Health Hered 2012-2012, 2012. 